\title{
PAPR Reduction in MIMO-OFDM System using Resource Block Technique
}

\author{
Jyoti M.Chavan ${ }^{1}$, S. K. Sudhansu ${ }^{2}$ \\ ${ }^{1,2}$ P. G. Dept., MBES College of Engineering, Ambajogai, India, 431517
}

\begin{abstract}
MIMO-OFDM system is fast growing new wireless broadband technology which has capability of high data rate transmission and advantages like inter carrier interference (ICI) reduction; high reliability; and better performance in multi-path fading. The major effect to be considered at receiver is fading effects which must be mitigated at receiver using equalization technique. In this paper we presented PAPR reduction in MIMO-OFDM with resource block, when it is compared with existing PAPR reduction techniques such as conventional PTS, selected mapping, clipping and filtering. The simulation shows PAPR reduction.
\end{abstract}

Keywords: Multiple input multiple output (MIMO), orthogonal frequency division multiplexing (OFDM), Partial Transmit Sequence (PTS), Space Frequency Block Coding (SFBC).

\section{Introduction}

Today wireless communication plays important role in our day to day life since most of the task can be done on multiple inputs multiple outputs (MIMO). Orthogonal frequency division multiplexing (OFDM) is a successful technology for high data rate transmission. Advantages of OFDM technique are high spectral efficiency, low implementation complexity and nonlinear distortion. But there is problem in OFDM that is high peak to average power ratio (PAPR).

The most challenging is in OFDM is to reduce PAPR, there are various methods, some of these are summarized in [2].One of the technique to reduce PAPR is PTS scheme which can achieve close PAPR reduction performance over the conventional PTS scheme if the threshold value is selected appropriately [3]. In some of the techniques of PAPR reduction, the candidate signal pairs of the two antennas are generated in the time-domain, rather than the frequency domain, for complexity reduction by using a variety of time-domain signal properties inherent in SFBC MIMO-OFDM systems [5]. The general formulas of the proposed scheme are derived and the trade-off between PAPR reduction and BER performance is achieved by setting the value of a parameter [6]. In the TR scheme, a small number of unused subcarriers called peak reduction carriers (PRCs) are reserved to reduce the PAPR and the goal of the TR scheme is to find the optimal values of the PRCs that minimize the PAPR of the transmitted OFDM signal [7]. Here develop two novel SLM schemes with much lower complexity than the conventional one; the first method uses only one IFFT block to generate the set of candidate signals, while the second one uses two IFFT blocks [8]. A complex orthogonal design that provides full diversity and full transmission rate for a space-time block code is not possible for more than two antenna [9].

\section{Proposed Method}

In SFBC MIMO OFDM system with two transmitting antennas, the input data block is encoded with the Alamouti space frequency encoder into two vectors, as follows[1]:

$$
\begin{array}{r}
\mathrm{X}_{1}=\left[\mathrm{X}_{0},-\mathrm{X}_{1}{ }^{*} \ldots \mathrm{X}_{\mathrm{N}-2},-\mathrm{X}_{\mathrm{N}-1}{ }^{*}\right]^{\mathrm{T}} \\
\mathrm{X}_{2}=\left[\mathrm{X}_{1}, \mathrm{X}_{0}{ }^{*} \ldots \mathrm{X}_{\mathrm{N}-1}, \mathrm{X}_{\mathrm{N}-2}{ }^{*}\right]^{\mathrm{T}}
\end{array}
$$

where $\mathrm{N}$ is the number of subcarrier and (.)*denotes the complex conjugate operation [10]. The continuous time baseband OFDM signal of $X_{i}$ for $i=1,2$ can be represented as [1]

$$
\mathrm{x}_{\mathrm{i}}(\mathrm{t})=\frac{1}{\sqrt{N}} \sum_{\mathrm{K}=0}^{\mathrm{N}-1} X_{\mathrm{i}, \mathrm{e}} e^{j 2 \Pi \Delta f t}, 0 \leq t \leq T
$$

where $\mathrm{X}_{\mathrm{i}, \mathrm{k}}$ represents the data symbol modulated by the kth subcarrier for $\mathrm{k}=0,1, \ldots \mathrm{N}-1$ in transmitting antenna $\mathrm{T}_{\mathrm{Xi}}$ with $\mathrm{i}=1,2, \Delta \mathrm{f}$ is the frequency difference between subcarriers, and $\mathrm{T}$ is the OFDM symbol duration. The PAPR of $x_{i}(t)$ in (2) is defined as the ratio of the maximum power to the average power of $x_{i}(t)$ and is expressed as [1]

$$
\operatorname{PAPR}_{i}=\frac{\max _{0 \leq t \leq T}|x i(t)|^{2}}{E\left[|x i(t)|^{2}\right]}
$$


where $\mathrm{E}[\cdot]$ denotes the expected value operation. The discrete time OFDM signal $\mathrm{x}_{\mathrm{i}}=\left[\mathrm{x}_{\mathrm{i}, 0}, \mathrm{x}_{\mathrm{i}, 1, \ldots \ldots . .} \mathrm{x}_{\mathrm{i}, \mathrm{N}-1}\right]^{\mathrm{T}}$ in antenna $T_{\mathrm{Xi}}$ is usually generated by sampling $\mathrm{x}_{\mathrm{i}}(\mathrm{t})$ with the rate $\mathrm{N} / \mathrm{T}$ and can be expressed as[1]

$$
\mathrm{x}_{\mathrm{i}, \mathrm{n}}=\frac{1}{\sqrt{N}} \sum_{\mathrm{k}=0}^{\mathrm{N}-1} \mathrm{x}_{\mathrm{i}, \mathrm{k}} e^{\frac{j 2 \pi k n}{N}}, \mathrm{n}=0,1, \ldots \ldots \ldots \ldots . \mathrm{N}-1
$$

FromTx $x_{1}$ and $\mathrm{Tx}_{2}$ antennas, $\mathrm{x}_{1}$ and $\mathrm{x}_{2}$ signals are transmitted. The PAPR of these two signal $\left(\mathrm{x}_{1}\right.$ and $\left.\mathrm{x}_{2}\right)$ can be calculated as:

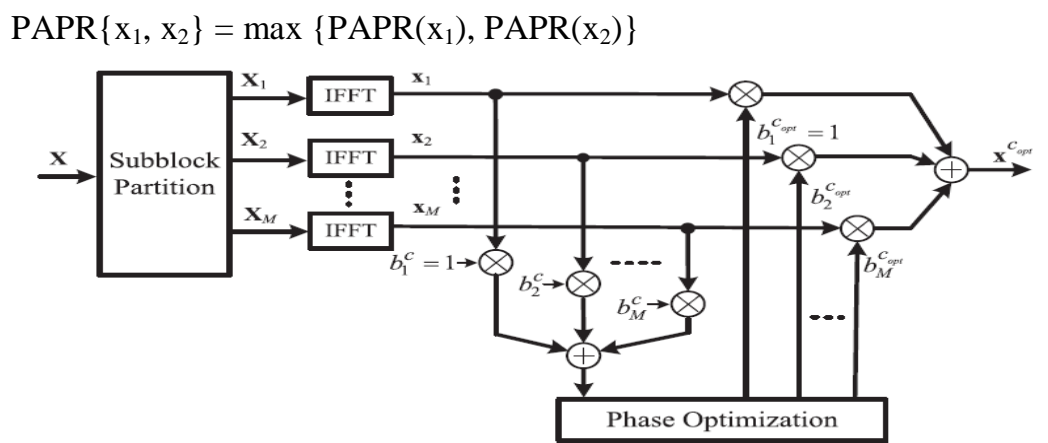

Fig.1. Block diagram of the conventional PTS scheme [1].

Above Fig.1 shows conventional Partial Transmit Sequence (PTS) scheme. For PAPR reduction PTS technique is used. For In the type I PTS scheme, the conventional PTS scheme is applied to $\mathrm{Tx}_{1}$ and $\mathrm{Tx}_{2}$ antennas. The data block $X_{i}$ of (1) is divided into $M_{b}$ disjoint subblocks. IFFT is used to convert frequency domain to time domain. The IFFT output of each subblocks are multiplied by a rotation factor, and then add together to form a candidate signal. The phase factors are selected to minimize the PAPR of $\mathrm{x}_{\mathrm{i}}(\mathrm{t})$.

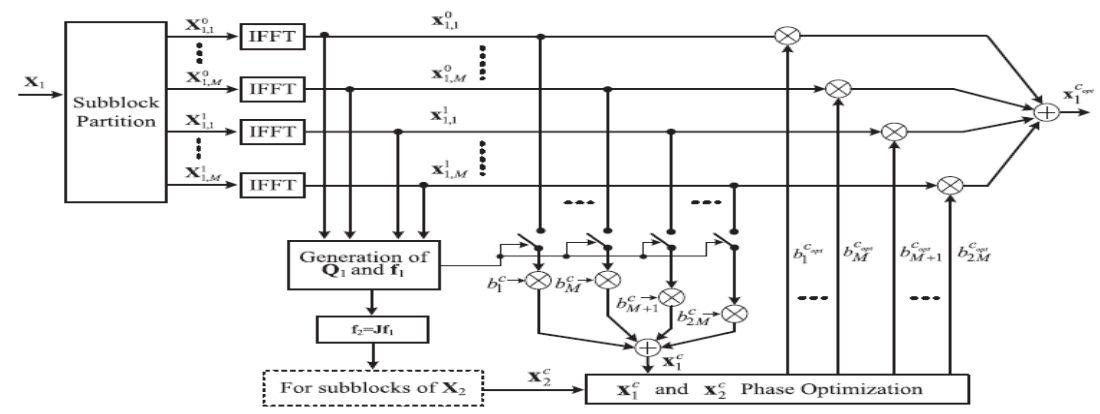

Fig.2. Part of the block diagram of type II PTS scheme for $\mathrm{T}_{\mathrm{x} 1}[1]$

Above Fig. 2 shows the type II PTS scheme. $\mathrm{X}_{1}$ and $\mathrm{X}_{2}$ signals are decomposed into even and odd parts as:

$$
\begin{aligned}
& \mathrm{X}_{1}^{0}=\left[\mathrm{X}_{0}, 0, \mathrm{X}_{2} \ldots \mathrm{X}_{\mathrm{N}-2}, 0\right]^{\mathrm{T}} \\
& \mathrm{X}_{1}^{1}=\left[0,-\mathrm{X}_{1}^{*}, 0 \ldots 0,-\mathrm{X}_{N-1}^{*}\right]^{\mathrm{T}} \\
& \mathrm{X}_{2}^{0}=\left[\mathrm{X}_{1}, 0, \mathrm{X}_{3} \ldots 0, \mathrm{X}_{\mathrm{N}-2}, 0\right]^{\mathrm{T}} \\
& \mathrm{X}_{2}^{1}=\left[0, \mathrm{X}_{0}^{*}, 0, \ldots \ldots, 0, \mathrm{X}_{N-2}^{*}\right]^{\mathrm{T}}
\end{aligned}
$$

It is clear $\mathrm{X}_{2}^{1}$ that can be obtained by performing shift-right and conjugate operations on $\mathrm{X}_{1}^{0}$, and that $\mathrm{X}_{2}^{0}$ can be obtained by performing shift-left, negate, and conjugate operations on $\mathrm{X}_{1}^{1}$. In addition, each of four signals of (6) is partitioned evenly into $\mathrm{M}$ subblocks. Each subblock is denoted as $\mathrm{X}_{i, k}^{e}$ with $\mathrm{e}=0,1, \mathrm{i}=1,2$, and $1 \leq \mathrm{m} \leq \mathrm{M}$. $\mathrm{Q}_{1}$ and $\mathrm{Q}_{2}$ are cost function and which are used to select samples for estimating peak power of candidate signals in each transmitting antennas. In above Fig.2 data $\mathrm{X}_{1}$ for antenna $\mathrm{Tx}_{1}$ in the SFBC MIMO-OFDM systems with two transmitting antennas. $f_{2}$ is used to select the samples to estimate the peak power of the candidate signals for antenna $\operatorname{Tx}_{2}$ [1]. In this paper Resource block is used in which the OFDM subcarriers are grouped into blocks and the phase of each block is changed same as the PTS technique by reducing the limitation of sending side information. 


\section{Simulations Result}

This paper analyses the bit error rate performance of MIMO OFDM using 16-QAM, 64-QAM as modulation schemes. Here a coded OFDM system with 64 subcarriers $(\mathrm{N}=64)$ and the number of constellations for QAM are 16, 64 i.e. 16-QAM, 64-QAM is considered. Matrix interleaver is used and cyclic prefix is set at $25 \%$ (16). There are two transmit and receive antennas. The figure (5-6) shows the BER Vs SNR for different systems using different modulation schemes.

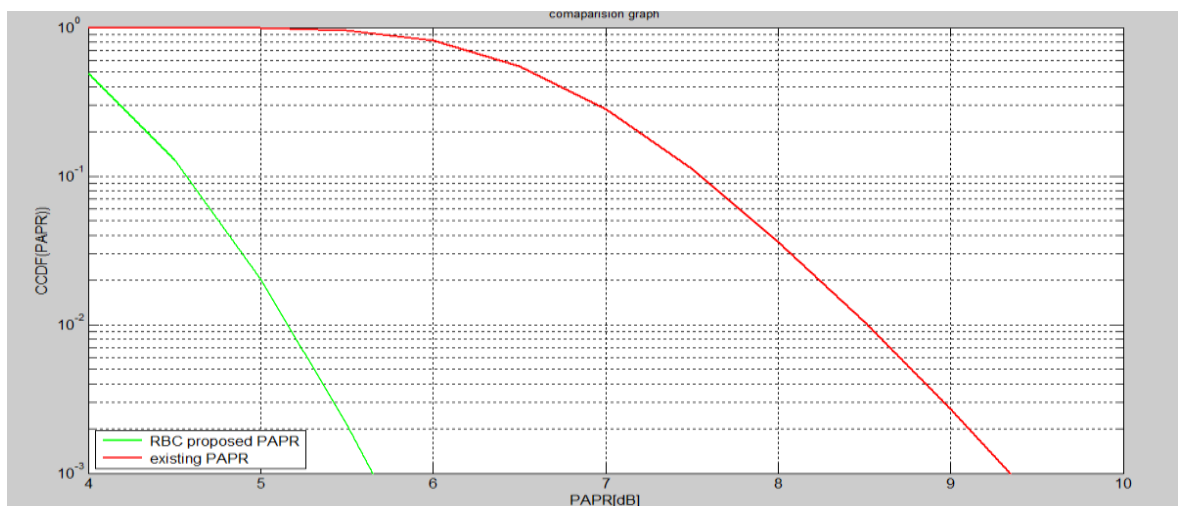

Fig.3. Comparison of PAPR reduction performance for existing method and resource block coding

Fig. 3 shows the comparison of PAPR reduction performance for existing method and resource block coding. By using resource block coding PAPR is reduced by $3.7 \mathrm{db}$. Resource blocks consisting of 12 subcarriers, which is referred as Physical Resource Block (PRB).Since subcarriers are separated by $15 \mathrm{kHz}$, the total bandwidth of a resource block is $(12 \times 15 \mathrm{KHz}=) 180 \mathrm{kHz}$. To demonstrate proposed MIMO OFDM, Bit error rate is presented in figure (4)-(5) and calculated for 16-QAM and 64-QAM modulation respectively.

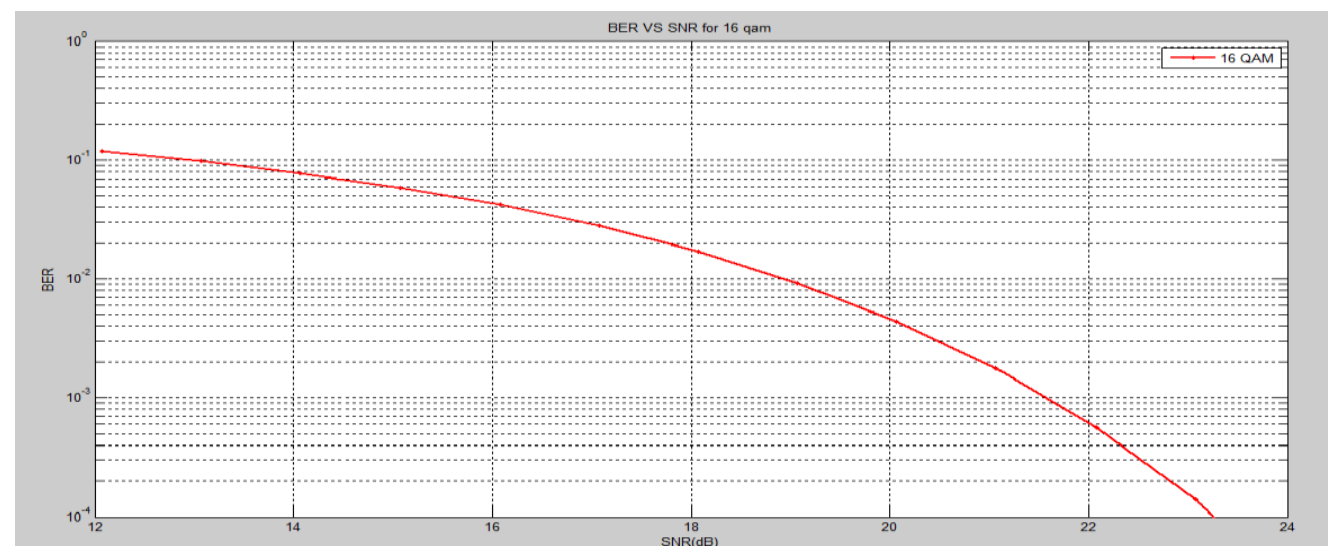

Fig.4. BER Vs SNR for 16-QAM

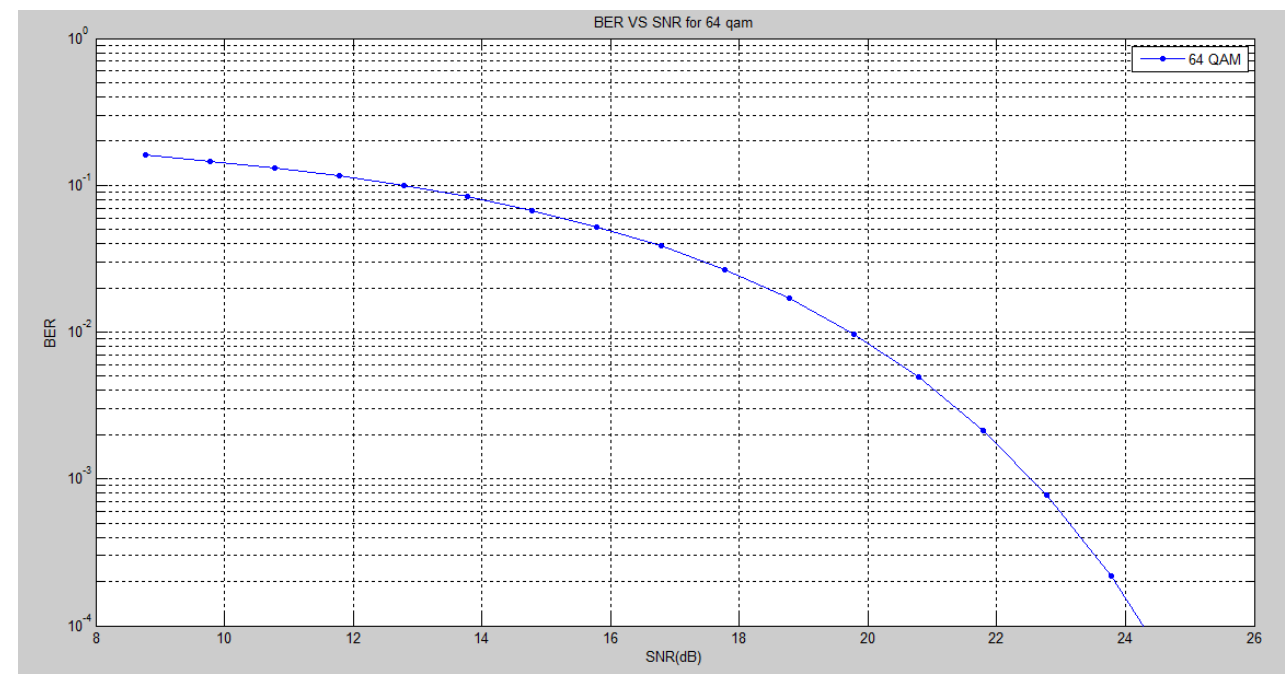

Fig.5. BER Vs SNR for 64-QAM 
Fig.4 and Fig.5 represents, BER Vs SNR for 16-QAM and 64-QAM modulation. It observes that, BER Vs SNR of $10^{-4}$ is achieved at 23dB and 24dB for 16-QAM and 64-QAM respectively, under AWGN channel. So from this graph it is observed that 16 QAM is better than 64 QAM. This is because for the same value of SNR , BER is reduced in 16 QAM.

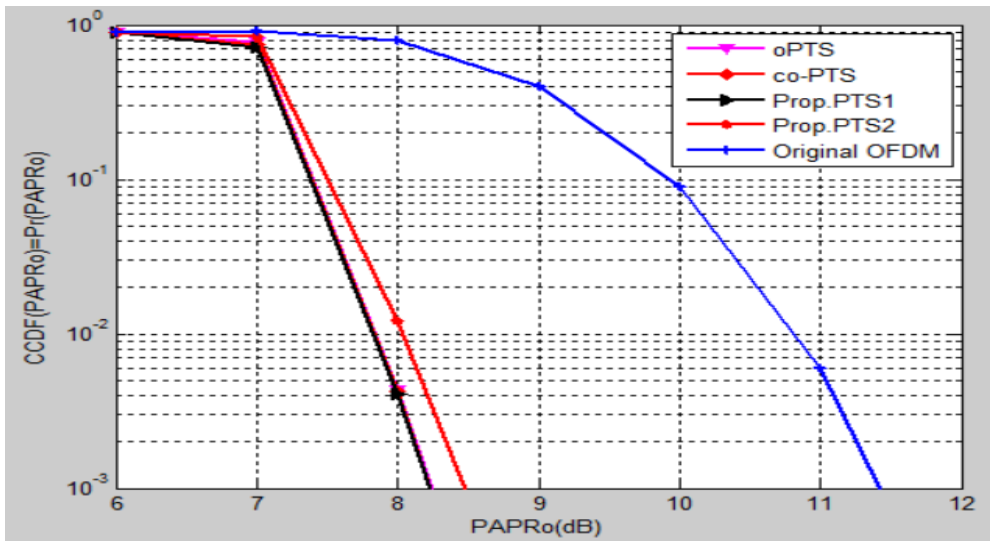

Fig.6. Comparison of PAPR reduction performance for the oPTS, the co-PTS, and the proposed type I PTS scheme [1]

Figs. 6-7 show the complementary cumulative distribution function (CCDF) of the PAPR for various PTS scheme in SFBC MIMO-OFDM system. Fig. 6 shows the PAPR reduction performance for the oPTS, the co-PTS, and the proposed type-I PTS scheme with different thresholds. Fig. 7 shows a comparison of PAPR reduction performance for the proposed type-II PTS scheme with different thresholds.

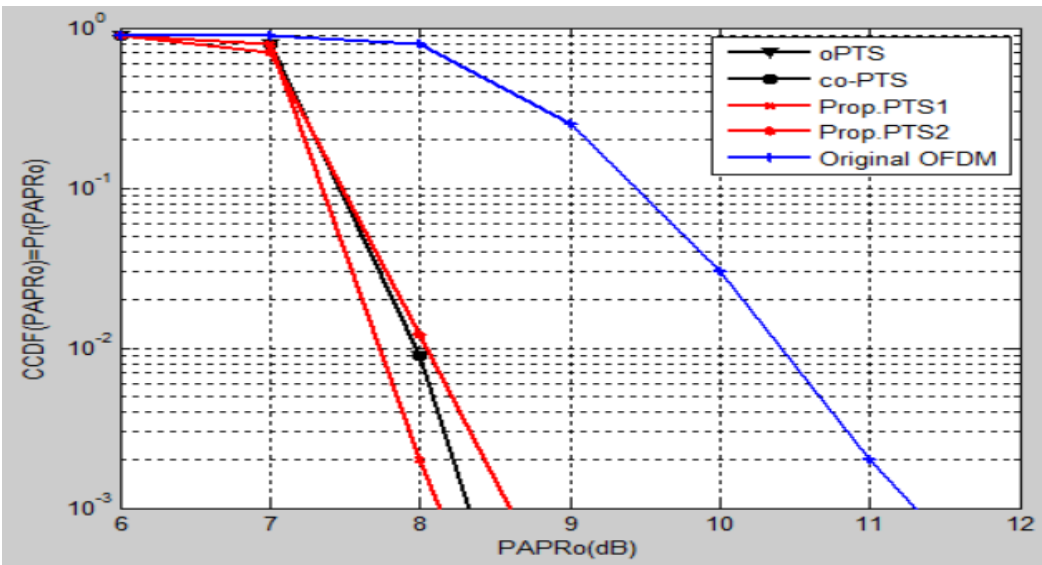

Fig.7. Comparison of PAPR reduction performance for the oPTS, the co-PTS, and the proposed type II PTS scheme [1]

\section{Conclusion}

In this paper, PTS technique with Resource Block Coding is proposed to reduce the PAPR of MIMOOFDM system. In type I and type II PTS has low computational complexity only because of using correlation among candidate signals. The simulation results showed that the proposed scheme could provide a better PAPR reduction performance than the SFBC MIMO-OFDM system by applying the PTS. In proposed system results show the BER Vs SNR graph for 16-QAM and 64-QAM modulation schemes over AWGN channel. Based on the simulation results it is concluded that by using 16-QAM scheme better BER performance for same value of SNR is obtained.

\section{Acknowledgements}

We convey our sincere thanks to the principal Dr. B. I. Khadakbhavi, dean of P.G. department Dr. V.G. Kasabegoudar and staff of MBES's College of Engineering, Ambajogai for help in carrying out this research work at the institute. 


\section{References}

[1]. Sheng-Ju Ku, “ Low-Complexity PTS-Based Schemes for PAPR Reduction in SFBC MIMO-OFDM Systems. IEEE Transactions on Broadcasting, VOL. 60, NO. 4, December 2014.

[2]. T. Jiang and Y.Wu, "An overview: Peak-to-average power ratio reduction techniques for OFDM signals," IEEE Trans. Broadcast., vol. 54, no. 2,pp. 257-268, Jun. 2008

[3]. S.-J. Ku, C.-L. Wang, and C.-H. Chen, “A reduced-complexity PTS-based PAPR reduction scheme for OFDM systems," IEEE Trans.Wireless Commun., vol. 9, no. 8, pp. 2455-2460, Aug. 2010.

[4]. J. Hou, J. Ge, and J. Li, "Peak-to-average power ratio reduction of OFDM signals using PTS scheme with low computational complexity,"IEEE Trans. Broadcast., vol. 57, no. 1, pp. 143-148, Mar. 2011.

[5]. S.-H. Wang and C.-P.Li, “A low-complexity PAPR reduction scheme for SFBC MIMO-OFDM systems,” IEEE Signal Process. Lett., vol. 16 no. 11, pp. 941-944, Nov. 2009.

[6]. S. S. Jeng and J. M. Chen, "Efficient PAPR reduction in OFDM systems based on a companding technique with trapezium distribution," IEEE Trans. Broadcast., vol. 57, no. 2, pp. 291-298, Jun. 2011.

[7]. J.-C. Chen, M.-H. Chiu, Y.-S. Yang, and C.-P. Li, "A suboptimal tone reservation algorithm based on cross-entropy method for PAPR reduction in OFDM systems," IEEE Trans. Broadcast., vol. 57, no. 3, pp. 752-756,Sep. 2011.

[8]. C.-L. Wang and Y. Ouyang, "Low-complexity selected mapping schemes for peak-to-average power ratio reduction in OFDM systems,” IEEE Trans. Signal Process., vol. 53, no. 12, pp. 4652-4660, Dec. 2005.

[9]. H. Jafarkhani, “A quasi-orthogonal space-time block code,” IEEE Trans Commun., vol. 49, no. 1, pp. 1-4, Jan. 2001.

[10]. S. M. Alamouti, “A simple transmit diversity technique for wireless communications,” IEEE J. Sel. Areas Commun., vol. 16, no. 8,pp. 1451-1458, Oct. 1998. 